\title{
Análisis epidemiológico de la diarrea en Santander y Norte de Santander
}

\section{Epidemiological analysis of diarrhea in Santander and Norte de Santander}

\author{
María del Pilar Gallardo-Lizarazo ${ }^{1}$
}

Forma de citar: María del Pilar Gallardo-Lizarazo. Análisis epidemiológico de la diarrea en Santander y Norte de Santander. Eco.Mat. 2015; 6(1): 78-85.

Recibido:

Junio 22 de 2015

Aceptado:

Agosto 10 de 2015

\section{Resumen}

La enfermedad diarreica aguda (EDA), a nivel mundial, se encuentra establecida como la segunda causa de muerte, afectando principalmente a los menores de 5 años sin desconocer los demás grupos cronológicos y sin distinguir características étnicas. En Colombia ocupa el segundo lugar de morbi-mortalidad en la población menor de cinco años, especialmente en los municipios con mayor porcentaje de necesidades básicas insatisfechas; por ello se han identificado estrategias para educar a las madres o cuidadores sobre el manejo adecuado en casa y centros de salud de primer nivel buscando la oportuna atención y realización de las convenientes prácticas de hidratación, para lo cual se creó la estrategia de Atención Integrada de las Enfermedades Prevalentes de la Infancia (AIEPI) que está encaminada en disminuir la mortalidad por las enfermedades prevalentes de la infancia específicamente en menores de 5 años. En el presente artículo se pretende describir la situación epidemiológica de EDA en los departamentos de Santander y Norte de Santander así como de las creencias, costumbres y conocimientos en madres y cuidadores sobre los signos de alarma en EDA en niños menores de 5 años de edad.

Palabras Claves: Enfermedad Diarreica Aguda, Morbilidad, Cuidado
${ }^{1}$ Médico General, Universidad de Santander. Estudiante Especialización en Epidemiología, Universidad Autónoma de Bucaramanga. mapygallar88@gmail.com

\begin{abstract}
Acute diarrheal disease (ADD), globally, is established as the second cause of death, affecting mainly children under 5 years without ignoring the chronological other ethnic groups without distinguishing characteristics. In Colombia it ranks second in morbidity and mortality in the population under five years, especially in the municipalities with the highest percentage of unmet basic needs; therefore they have identified strategies to educate mothers and caregivers about the proper management at home and health centers looking for first-rate care and timely implementation of appropriate hydration practices, for which the strategy of Integrated Management was established Prevalent Childhood Illness (IMCI) that aims to reduce mortality from prevalent childhood diseases specifically in children under 5 years. In the present article is to establish the epidemiological situation of ADD in the departments of Santander and Norte de Santander and beliefs, habits and knowledge among mothers and caregivers about the signs of alarm in ADD in children under 5 years of age.
\end{abstract}

Keywords: Acute Diarrheal Disease, Morbidity, Care 


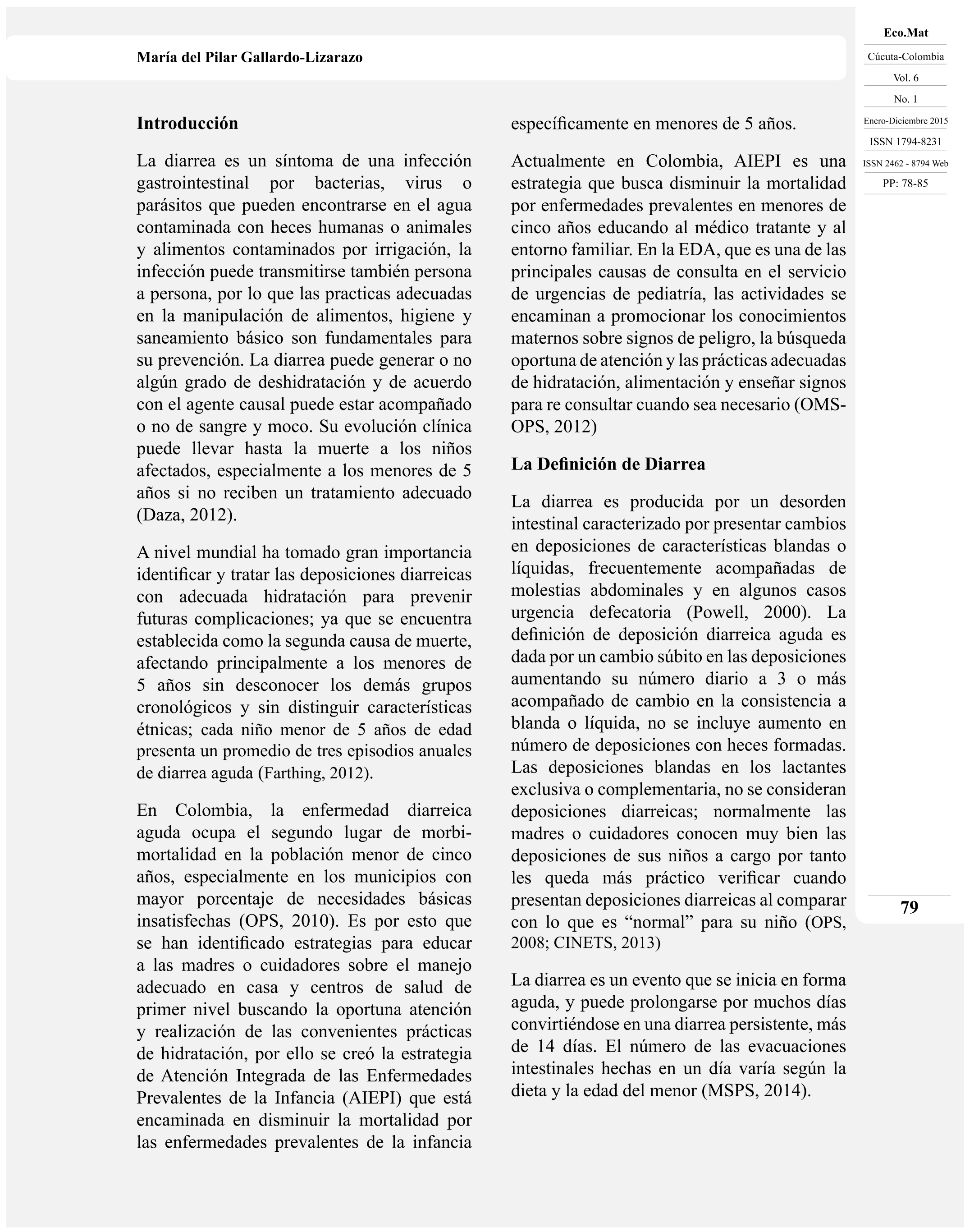




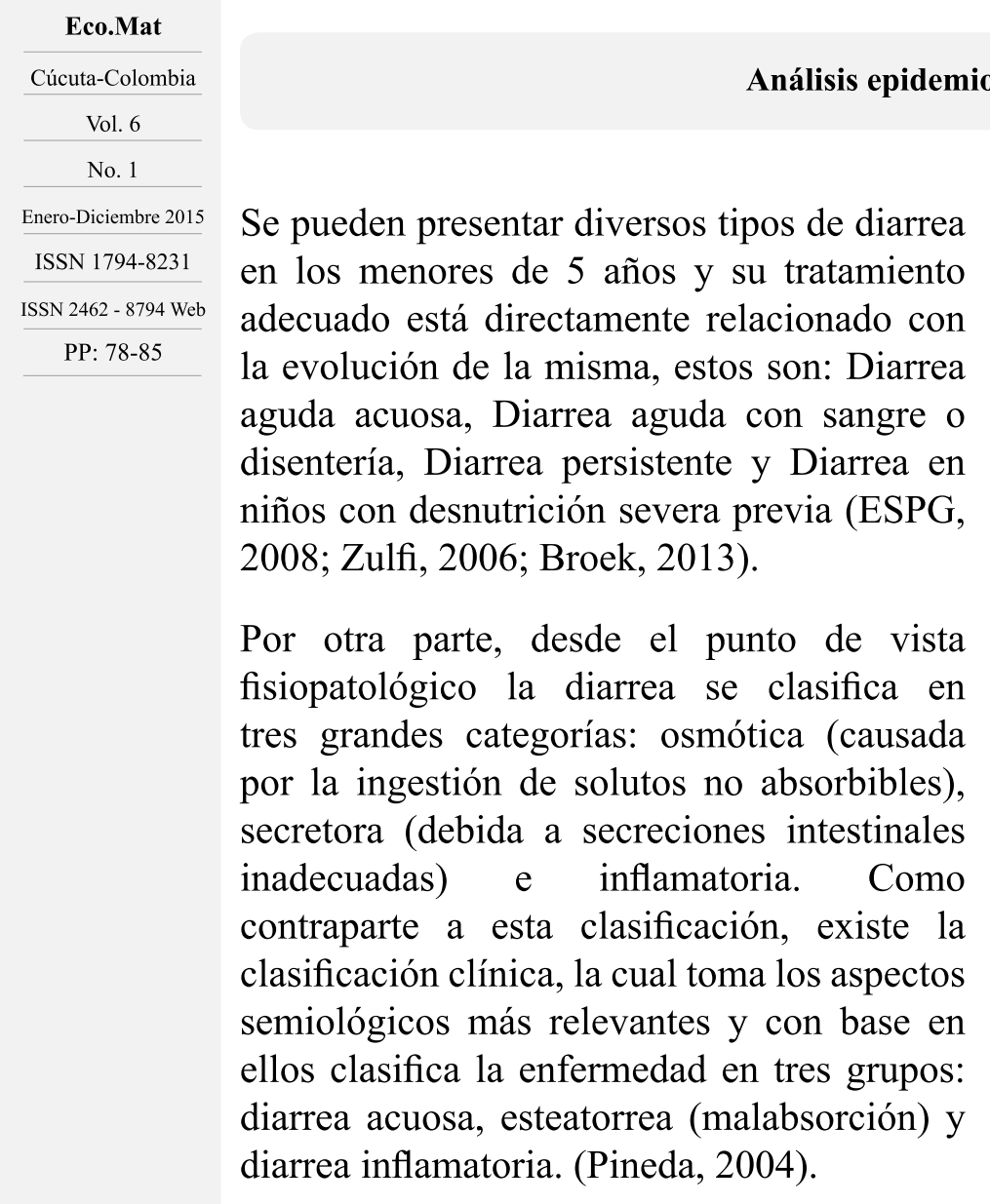

\section{Caracterización de la Población.}

El Departamento de Santander cuenta con una población de 2.061.095 habitantes, según la proyección del DANE a 2015, con una población femenina de 50,6\% frente al 49,4\% de la masculina y una tasa de crecimiento poblacional de $0,50 \%$. Su población constituye un $4,30 \%$ de la población nacional. El 75,4\% de su población se ubica en cabeceras municipales; la ciudad de Bucaramanga, su capital, concentra el $25,6 \%$ de la población del departamento. Cuatro ciudades importantes, Floridablanca, Barrancabermeja, Girón y Piedecuesta, concentran un $38,2 \%$ y los 82 municipios restantes, el $36,2 \%$ de la población, entre los que se encuentran San Gil, El Socorro, Lebrija y Barbosa

El departamento Norte de Santander cuenta con una población de 1.355.787 habitantes, según la proyección del DANE a 2015, con una población femenina de 50,4\% frente al $49,6 \%$ de la masculina y una tasa de crecimiento poblacional de $0,86 \%$. $\mathrm{Su}$ población constituye un $2,82 \%$ de la población nacional. El 78,5\% de la población se ubica en cabeceras municipales; la ciudad de Cúcuta, su capital, concentra el $48 \%$ de la población del departamento. Cuatro ciudades importantes, Ocaña, Villa del Rosario, Los Patios y Pamplona, concentran un $17 \%$ y los 35 municipios restantes, el $35 \%$ de la población.

En la figura 1 se presentan las pirámides poblacionales para los dos departamentos; se aprecia mayor concentración en población de menor edad para el Norte de Santander, pero con un decrecimiento más acentuado a medida que se avanza en cohortes de edad que en Santander.

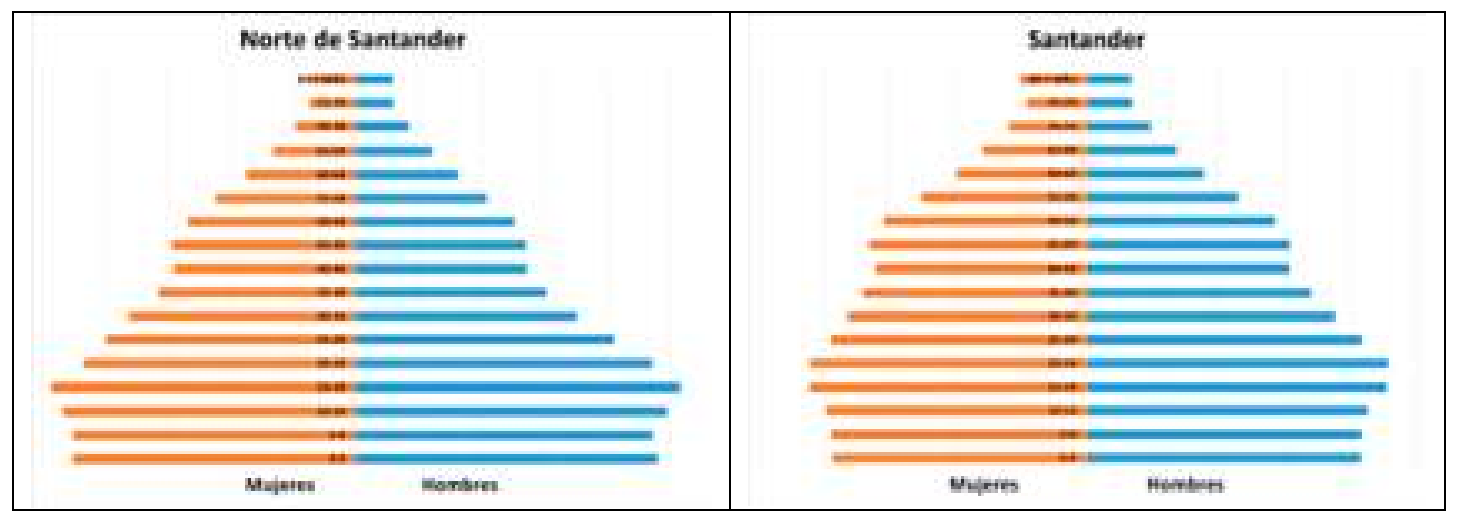

Figura 1: Distribución de la Población por Cohortes de Edad, proyección a 2015 elaborada por la autora con base en datos del DANE 


\section{María del Pilar Gallardo-Lizarazo}

El $8,0 \%$ de la población de Santander es menor de cinco años, distribuida en 51,3\% niños y 48,3\% niñas; en Bucaramanga la proyección a 2015 indica un total de 34.922 , sin embargo, al incluir los municipios del área metropolitana (Floridablanca, Girón y Piedecuesta) se obtiene un total de 79.609 que constituye el $63 \%$ de niños y niñas menores de cinco años del departamento.

Por otra parte, el 9,3\% de la población de Norte de Santander es menor a cinco años con 51,1 \% niños y 48,9 niñas; en Cúcuta la proyección a 2015 indica un total de 55.993, sin embargo, al incluir los municipios del área metropolitana (Villa del Rosario, Los Patios, El Zulia y san Cayetano) se obtiene un total de 73.332 que constituye el $58 \%$ de niños y niñas menores de cinco años del departamento.

\section{Mortalidad y Morbilidad por EDA}

El Instituto Nacional de salud indica que en Colombia más de 250.000 niños mueren antes de los 5 años por enfermedades que podrían prevenirse fácilmente. Estas muertes ocurren principalmente por diarrea, neumonía, desnutrición, y otras enfermedades prevenibles por vacunación, siendo estas enfermedades la causa de 60 a $80 \%$ de las consultas pediátricas en los servicios de salud y de 40 a $50 \%$ de las hospitalizaciones de niños menores de 5 años. (Álvarez, 2014). La mortalidad por enfermedad diarreica aguda ocupa uno de los primeros lugares en la mortalidad de los menores de 5 años especialmente en los municipios con mayor proporción de necesidades básicas insatisfechas y mala calidad de agua para consumo humano (Borbón, 2013).
Eco.Mat

Cúcuta-Colombia Vol. 6

No. 1

Enero-Diciembre 2015 ISSN 1794-8231 ISSN 2462 - $8794 \mathrm{Web}$ PP: $78-85$

Tabla I. MORTALIDAD POR EDA EN MENORES DE 5 AÑOS

\begin{tabular}{|c|c|c|c|c|c|c|}
\hline \multirow{2}{*}{ Año } & \multirow{2}{*}{$\begin{array}{c}\text { Muertes } \\
\text { por EDA en } \\
<5 \text { años }\end{array}$} & \multicolumn{2}{|c|}{$\begin{array}{c}\text { Incidencia por millón de } \\
\text { habitantes en Colombia }\end{array}$} & \multicolumn{2}{|c|}{$\begin{array}{c}\text { Incidencia por millón de } \\
\text { habitantes [<5 años] }\end{array}$} \\
\cline { 4 - 7 } & $<\mathbf{1}$ año & $\begin{array}{c}\mathbf{1} \text { a } \mathbf{4} \\
\text { años }\end{array}$ & $<\mathbf{5}$ años & Santander & $\begin{array}{c}\text { Norte de } \\
\text { Santander }\end{array}$ \\
\hline $\mathbf{2 0 1 1}$ & 138 & 8,12 & 1,99 & 3,22 & 5,90 & 46,90 \\
\hline $\mathbf{2 0 1 2}$ & 109 & 70,50 & 14,01 & 26,33 & 5,93 & 31,49 \\
\hline $\mathbf{2 0 1 3}$ & 117 & 76,1 & 14,9 & 27,20 & 11,90 & 15,80 \\
\hline $\mathbf{2 0 1 4}$ & 149 & 89,6 & 20,6 & 34,57 & 5,96 & 15,80 \\
\hline
\end{tabular}

Elaborada por la autora con datos de DANE y SIVIGILA

En la tabla I se presenta el número de casos y la incidencia acumulada de mortalidad por EDA en menores de 5 años por cada millón de habitantes a semana epidemiológica 52 del año, se aprecia un crecimiento en la incidencia a lo largo del tiempo, sin embargo, la tasa es menor en Santander que en Norte de Santander. El porcentaje de muertes es mayor en el género masculino, con un promedio anual de $56 \%$, como se muestra en la figura 2. La mayor notificación de casos se presenta en población afiliada al sistema de seguridad social en salud al régimen subsidiado, le siguen los no afiliados y en menor proporción se encuentran los casos del régimen contributivo, esta tendencia se mantiene en el tiempo. Al desagregar por lugar de residencia se encuentra que en los últimos años más de la mitad de la población atendida reside en el sector rural (ver tabla II).

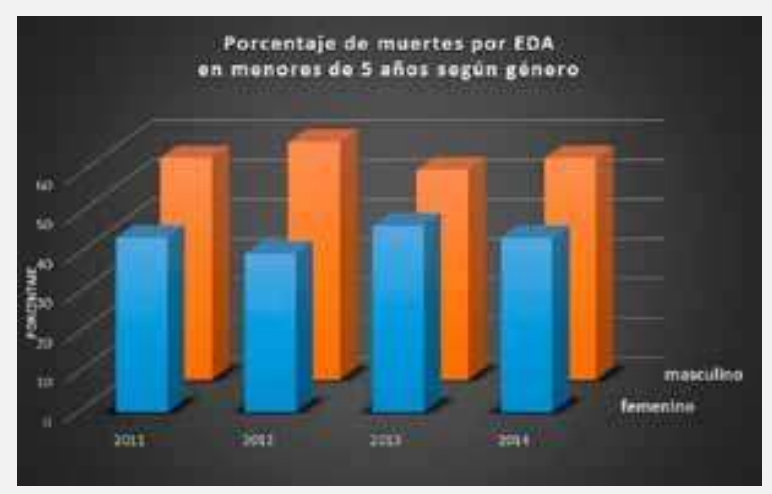

Figura 2. Mortalidad por EDA según género en menores de 5 años Elaborada por la autora con datos de DANE y SIVIGILA 


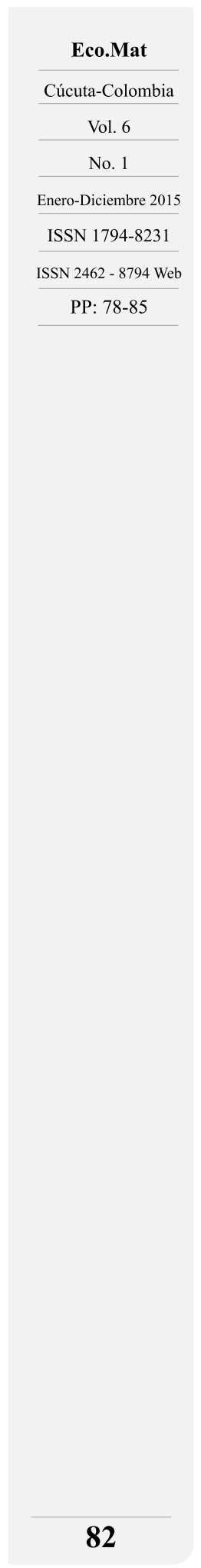

Análisis epidemiológico de la diarrea en Santander y Norte de Santander

TABLA II. ATENCIÓN POR SISTEMA DE SEGURIDAD Y POR LUGAR DE PROCEDENCIA

\begin{tabular}{|c|c|c|c|c|c|c|}
\hline \multirow{2}{*}{ Año } & \multicolumn{2}{|c|}{$\begin{array}{c}\text { Porcentaje atendido por sistema de } \\
\text { seguridad social en salud }\end{array}$} & \multicolumn{3}{c|}{$\begin{array}{c}\text { Porcentaje atendido por lugar de } \\
\text { residencia }\end{array}$} \\
& $\begin{array}{c}\text { Régimen } \\
\text { subsidiado }\end{array}$ & $\begin{array}{c}\text { Régimen } \\
\text { contributivo }\end{array}$ & No afiliado & $\begin{array}{c}\text { Cabecera } \\
\text { municipal }\end{array}$ & $\begin{array}{c}\text { Centro } \\
\text { poblado }\end{array}$ & $\begin{array}{c}\text { Rural } \\
\text { disperso }\end{array}$ \\
\hline 2011 & 59 & 11 & 30 & 35 & 12 & 53 \\
\hline 2012 & 70 & 9 & 21 & 49 & 15 & 36 \\
\hline 2013 & 68 & 6 & 26 & 40 & 10 & 50 \\
\hline 2014 & 69 & 7 & 24 & 31 & 13 & 56 \\
\hline
\end{tabular}

Elaborada por la autora con datos de DANE y SIVIGILA

Para el análisis de la morbilidad por EDA se recurre a la información en el sistema de vigilancia del Instituto Nacional de Salud con base en los reportes para este evento por parte de las entidades territoriales. La incidencia de morbilidad por EDA en el país, para el año 2014 , es de 60,05 por cada mil habitantes, más alta en comparación con la de 2013 que fue de 57,3 y de 54,76 en 2012 (Daza, 2012).

\begin{tabular}{|c|c|ccc|}
\hline \multirow{2}{*}{ Año } & $\begin{array}{c}\text { Morbilidad por } \\
\text { EDA en < 5 años }\end{array}$ & \multicolumn{3}{|c|}{$\begin{array}{c}\text { Incidencia por 1000 } \\
\text { habitantes en Colombia }\end{array}$} \\
\cline { 2 - 5 } & Casos reportados & $<\mathbf{1}$ año & $\begin{array}{c}\mathbf{1} \text { a 4 } \\
\text { años }\end{array}$ & $<\mathbf{5}$ años \\
\hline $\mathbf{2 0 1 1}$ & 728879 & 254,6 & 148,8 & 170,1 \\
\hline $\mathbf{2 0 1 2}$ & 714067 & 240,6 & 147,7 & 166,4 \\
\hline $\mathbf{2 0 1 3}$ & 669482 & 218,4 & 139,9 & 155,7 \\
\hline $\mathbf{2 0 1 4}$ & 681530 & 210,5 & 144,9 & 158,1 \\
\hline \multicolumn{4}{|c|}{ Elaborada por la autora con datos de DANE y SIVIGILA } \\
\hline
\end{tabular}

Con respecto al número de casos reportados de EDA (ver tabla III) y al comparados con los reportes nacionales para los diferentes grupos de edad se encuentra que en todos los grupos de edad se reportan casos pero la población menor de cinco años y en especial la población inferior a un año las que presentan tasas de incidencia más altas.

\begin{tabular}{|c|cc|cc|}
\multicolumn{4}{|c|}{ Tabla IV. Morbilidad por EDA en Santander y Norte de Santander } \\
\hline \multirow{3}{*}{ Año } & \multicolumn{4}{|c|}{ Morbilidad por EDA } \\
\cline { 2 - 5 } & \multicolumn{2}{|c|}{ Santander } & \multicolumn{2}{c|}{ Norte de Santander } \\
\cline { 2 - 5 } & $\begin{array}{c}\text { Casos } \\
\text { reportados }\end{array}$ & $\begin{array}{c}\text { Incidencia X } \\
\mathbf{1 0 0 0 0} \text { hab. }\end{array}$ & $\begin{array}{c}\text { Casos } \\
\text { reportados }\end{array}$ & $\begin{array}{c}\text { Incidencia X } \\
\mathbf{1 0 0 0 0} \text { hab. }\end{array}$ \\
\hline $\mathbf{2 0 1 1}$ & 79858 & 395 & 64197 & 490 \\
\hline $\mathbf{2 0 1 2}$ & 79101 & 389 & 61482 & 466 \\
\hline $\mathbf{2 0 1 3}$ & 74513 & 365 & 64370 & 483 \\
\hline $\mathbf{2 0 1 4}$ & 76960 & 375 & 68270 & 508 \\
\hline
\end{tabular}

Por otra parte, aun cuando Santander reporta más casos por año, la incidencia por EDA es menor en Santander que en Norte de Santander, sin embargo ambas son menores que la nacional (ver tabla IV y figura3), las entidades territoriales con mayor incidencia son Amazonas, Bogotá, Quindío y Meta. 


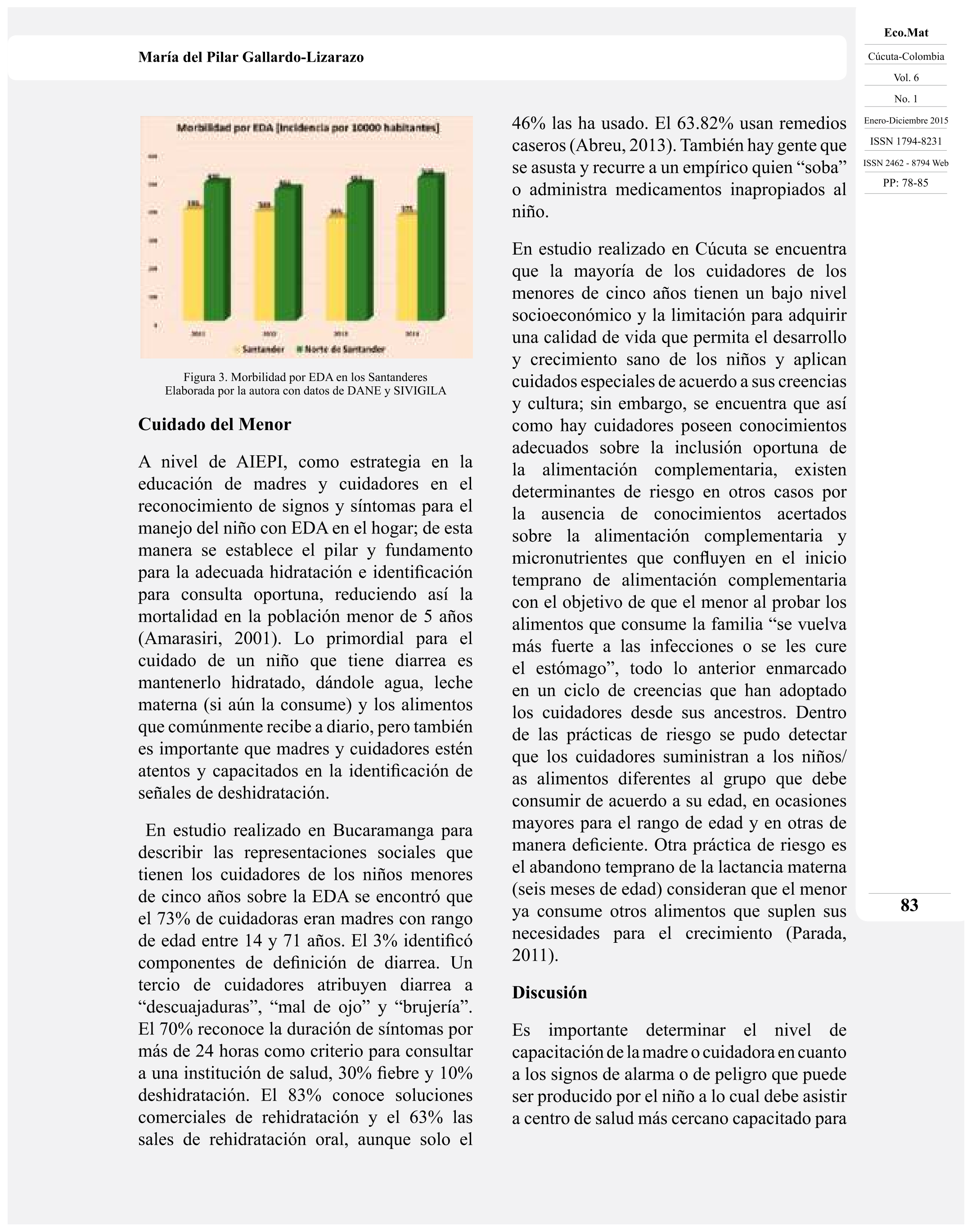




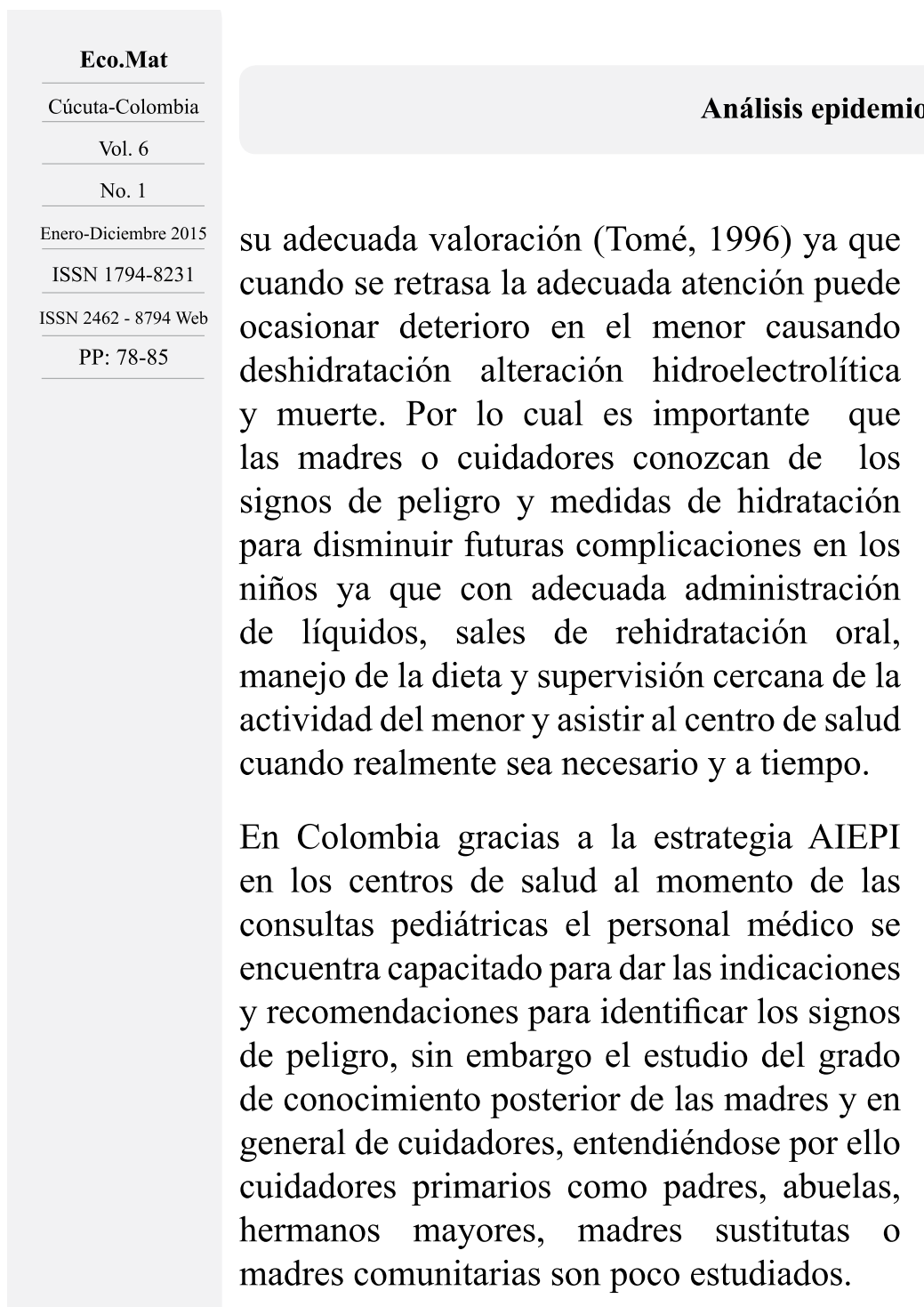

Al comparar cuidadores de niños con y sin episodio de diarrea, se encontró que los conocimientos sobre los signos y síntomas son mayores en el grupo con episodios, así como también la inclinación por remedios caseros, mientras que el conocimiento sobre la rehidratación oral es mayor en los cuidadores de niños que no los presentaron. En conclusión, la investigación identificó falta de conocimientos de cuidadores al reconocer episodios de EDA y signos de alarma; también concluyó que a pesar del conocimiento en fórmulas comerciales y en SRO, la mayoría de cuidadores prefiere, reemplazar o adicionar al manejo remedios caseros (Abreu 2013),

Es conveniente continuar realizando estudios en los departamentos de Santander y Norte de Santander sobre creencias, actitudes y conocimientos de los cuidadores de niños con respecto a la diarrea, así se pueden canalizar esfuerzos en capacitación para su identificación, atención y consulta oportuna con miras a disminuir sus tasas de morbilidad y mortalidad

\section{Referencias Bibliográficas}

Álvarez, C. y Equipo Funcional ETA. (2014). Informe Final Mortalidad por Enfermedad Diarreica Aguda en Menores de Cinco Años y Morbilidad en Todos los Grupos de Edad, Colombia, 2014. Instituto Nacional de Salud, Subdirección de Vigilancia y Control de Salud Pública.

Abreu Peralta, P. y otros. (2013). Conocimientos, Creencias y Prácticas en el Cuidado de la Diarrea Aguda en Hogares de la Zona Norte de Bucaramanga, Santander 2013 - ResearchGate.

Amarasiri de Silva MW, Wijekoon A, Hornik R, Martines J. (2001). Care seeking in Sri Lanka: one possible explanation for low childhood mortality. Soc Sci Med 2001; 53: 1363-1372.

Borbón, M. (2013). Mortalidad por Enfermedad Diarreica Aguda en Menores de Cinco Años y Morbilidad en Todos los Grupos de Edad, Colombia, año 2013. Instituto Nacional de Salud, Subdirección de Vigilancia y Control de Salud Pública.

Broek, I y otros. (2013) Guía Clínica y Terapéutica. Médicos sin Fronteras.

CINETS. (2013). Guía de Práctica Clínica para Prevención, Diagnóstico y Tratamiento de la Enfermedad Diarreica Aguda en Niños Menores de 5 años. MinSalud-Colciencias. Bogotá

Daza, J. y Grupo EDA. (2011). Informe del evento mortalidad por enfermedad diarreica aguda en menores de cinco años, y morbilidad en todos los grupos de edad, año 2011. Instituto Nacional de Salud, Subdirección de 
Vigilancia y Control de Salud Pública.

Daza, J. y Grupo EDA-IRA. (2012). Informe del evento mortalidad por enfermedad diarreica aguda en menores de cinco años, y morbilidad en todos los grupos de edad, año 2012. Instituto Nacional de Salud, Subdirección de Vigilancia y Control de Salud Pública.

DANE. Estadísticas en Salud:

https://www.google.com.co/?gfe_rd=cr\&ei= XpPtVanPBYKw8we8laLgDQ\&gws_rd=ssl $\# \mathrm{q}=$ MSPS + dane + estad $\%$ C3\%ADsticas + en+ salud

DANE. Estadísticas en Salud, Estadísticas Vitales: http://www.dane.gov.co/index.php/ poblacion-y-registros-vitales/nacimientos-ydefunciones/nacimientos-y-defunciones

ESPG. (2008). European Society for Pediatric Gastroenterology, Hepatology, and Nutrition / European Society for Pediatric Infectious Diseases Evidence-based Guidelines for the Management of Acute Gastroenteritis in Children in Europe. JPGN 46:S81-S122, 2008.

Farthing, M. y otros. (2012). Diarrea Aguda en Adultos y Niños: una Perspectiva Mundial. Guía Práctica de la Organización Mundial de Gastroenterología. Reino Unido.

SIVIGILA. Instituto Nacional de Salud Sistema Nacional de Vigilancia en Salud Pública:

OMS, OPS (2012). Manual de atención integrada a las enfermedades prevalentes de la infancia. Washington, DC: OMS/OPS.

OPS. (2008). Tratamiento de la diarrea: Manual Clínico para los Servicios de Salud. Washington, D.C.

OPS. (2010). Atención Integrada a las Enfermedades Prevalentes de la Infancia: Libro Clínico. Bogotá. www.paho.org/col

Parada, D. (2011). Conocimientos, actitudes y prácticas del cuidador del menor de cinco años. Revista Ciencia y Cuidado. Vol. 8. No. 1: 29-39.

Pineda, L., W. Otero y V. Arbeláez. (2004). Diarrea Crónica. Diagnóstico y Evaluación Clínica. Rev Col Gastroenterol, Apr./ June, vol.19, no.2. Bogotá

Powell D.W. (2000). Dianostic Aproach to Chorinc Diarrea. AGA posgraduate course volumen 2000-2: 13-21.

Tomé P, Reyes H, Rodríguez L, Guiscafré H, Gutiérrez G. (1996). Muerte por Diarrea Aguda en Niños: Un estudio de factores pronósticos. Salud Pública Mex; 38: 227-235.

Zulfi qar A. Bhutta. Diarrea persistente en países en vías de Desarrollo. Ann Nestlé [Esp] 2006; 64:39-48

http://www.ins.gov.co/lineas-de-accion/ Subdireccion-Vigilancia/sivigila/Paginas/ sivigila.aspx

MSPS. (2014). Protocolo de Vigilancia en Salud Pública. Mortalidad por Enfermedad Diarreica Aguda en $<5$ años (EDA). Ministerio de Salud y Protección Social. Instituto Nacional de Salud. Bogotá. 